\title{
DIRECT DESIGN OF ROBUSTLY ASYMPTOTICALLY STABILIZING HYBRID FEEDBACK
}

\author{
Rafal Goebel ${ }^{1}$ And Andrew R. TeEL ${ }^{2}$
}

\begin{abstract}
A direct construction of a stabilizing hybrid feedback that is robust to general measurement error is given for a general nonlinear control system that is asymptotically controllable to a compact set.
\end{abstract}

Mathematics Subject Classification. 93D15, 49J53, 93B35

Received April 24, 2007.

Published online March 6, 2008.

\section{INTRODUCTION}

Over the last decade, multiple approaches have emerged for constructing (discontinuous, in general) state feedback stabilizers for asymptotically controllable nonlinear systems. In [3], a feedback was constructed using locally Lipschitz control Lyapunov functions and nonsmooth calculus. The paper [1] introduced the notion of a patchy feedback, a state feedback that is constant on patches of the state space and gives the closed-loop vector field certain "inward pointing" properties on the boundary of a patch. Furthermore [1], showed that every asymptotically controllable nonlinear system admits a stabilizing patchy feedback. In both [3] and [1], the authors showed that the resulting feedbacks are robust to small additive disturbances. In [2], it was shown that the patchy feedbacks of [1] are robust, in the semiglobal practical sense, to measurement noise of small variation. However, robustness to general measurement noise could not be expected in general without modifications of the feedbacks of [3] and [1].

In [11] and [4], the feedback ideas in [3] were implemented, to guarantee some robustness to measurement noise, with sample and hold. Such implementation, where the control is held constant using a timer variable that is reset at each sampling instant, makes the resulting feedback hybrid. An alternative hybrid feedback idea is found in the notion of hysteresis switching. In the context of robust stabilization of nonlinear control systems, this approach was pursued initially in [7,8], using the patchy feedbacks of [1] as the supporting idea. In particular, robust stabilization of any asymptotically controllable nonlinear system was achieved in [8]. As general results on the robustness of asymptotic stability for hybrid systems were not available at the time, the robustness had to be established directly.

\footnotetext{
Keywords and phrases. Hybrid feedback, nonlinear control system, patchy feedback, robustness, asymptotic stability.

${ }^{1}$ Research carried out at the Center for Control, Dynamical Systems, and Computation; Department of Electrical and Computer Engineering; University of California, Santa Barbara.

Current address: 3518 NE 42 St., Seattle, WA 98105, USA. rafal.k.goebel@gmail.com.

2 Center for Control, Dynamical Systems, and Computation; Department of Electrical and Computer Engineering; University of California, Santa Barbara, CA 93106-9560, USA. teel@ece.ucsb.edu.
} 
In [5], various regularity properties of general hybrid systems were established and robustness of stability was tied to some basic properties of the system's data. The work in [9] adapted such results to nonlinear systems in closed loop with hybrid patchy feedbacks, and in particular, showed that any hybrid patchy feedback that is strongly regular (in the terminology of the current paper, see Def. 2.1 below) is robust with respect to external disturbances, actuator error, and measurement noise. Also in [9], a stabilizing and strongly regular hybrid patchy feedback was constructed from a stabilizing (not hybrid) patchy feedback, which exists for asymptotically controllable nonlinear systems according to [1]. Examples of hybrid patchy feedbacks, including those that robustly stabilize nonlinear systems that do not admit robustly stabilizing nonhybrid feedbacks (say, Artstein's circles, Brockett's integrator) can be found in [6,9].

In this note, we consider general asymptotically controllable nonlinear systems and give a direct construction of a stabilizing hybrid patchy feedback that is strongly regular. To an extent, we rely on the ideas of [1] and [9], but avoid the intermediate construction of a patchy vector field. As a result, we avoid the analysis needed in [1] to show the robustness of the patchy vector field to external disturbance - such robustness comes "for free", along with robustness to measurement noise, from the structure of the hybrid feedback and results of [9]. Since hybrid feedback is our goal, weaker properties are required from the objects corresponding to patches of [1] here, the vector fields need not be "inward-pointing" - and as a result, the construction is somewhat simpler.

\section{Preliminaries}

For an open set $\widetilde{\mathcal{O}} \subset \mathbb{R}^{n}$ and a compact set $\mathcal{A} \subset \widetilde{\mathcal{O}}$, consider a set of feasible controls $U \subset \mathbb{R}^{m}$, a function $f: \widetilde{\mathcal{O}} \times U \rightarrow \mathbb{R}^{n}$ and a nonlinear control system

$$
\dot{x}(t)=f(x(t), u(t)), \quad u(t) \in U, \text { for all } t \geq 0 .
$$

In what follows, we write $\mathcal{O}$ for $\widetilde{\mathcal{O}} \backslash \mathcal{A}$.

Definition 2.1. A hybrid patchy feedback consists of

- a set $Q$

- for each $q \in Q$,

- sets $C_{q} \subset \mathcal{O}$ and $D_{q} \subset \mathcal{O}$;

- a function $k_{q}: C_{q} \rightarrow U$;

- a set-valued mapping $G_{q}: D_{q} \rightrightarrows Q$.

A hybrid patchy feedback is regular if $Q \subset \mathbb{Z}^{\bar{n}}$ for some $\bar{n}$, and for each $q \in Q, C_{q}$ and $D_{q}$ are relatively closed subsets of $\mathcal{O}, k_{q}$ is continuous, $G_{q}$ is outer semicontinuous and locally bounded on $\mathcal{O}^{1}$, and $G_{q}(x)$ is nonempty for all $x \in D_{q}$. It is strongly regular if it is regular, $\left\{C_{q}\right\}_{q \in Q}$ form a locally finite covering of $O, G_{q}$ are locally bounded uniformly in $q$, and for all $q \in Q, C_{q} \cup D_{q}=\mathcal{O}$.

In closed loop with the nonlinear system (2.1), a hybrid patchy feedback as in Definition 2.1 leads to a hybrid system

$$
\begin{array}{ll}
\dot{x}=F_{q}(x) & \text { if } x \in C_{q}, \\
q^{+} \in G_{q}(x) & \text { if } x \in D_{q},
\end{array}
$$

on the state space $\mathcal{O} \times Q$, where for each $q \in Q, F_{q}(x):=f\left(x, k_{q}(x)\right)^{2}$.

\footnotetext{
${ }^{1}$ For these, and other concepts related to set-valued mappings, we point the reader to [10]. Here, we only note that $G_{q}$ is outer semicontinuous on $\mathcal{O}$ if and only if its graph is closed, relative to $\mathcal{O} \times \mathbb{Z}^{\bar{n}}$.

${ }^{2}$ The phrase "hybrid patchy feedback" was used previously in [8]. The feedback constructed there fits the current definition of a hybrid patchy feedback. We use the term hybrid patchy feedback, rather than just hybrid feedback, both to relate it to the patchy feedback of [1] and because hybrid feedback with different structure can be used for purposes of robust stabilization. In particular, the sample and hold implementation of the state feedback $u=\kappa(x)$ results in a hybrid system $\dot{x}=f(x, u), \dot{\tau}=1$ if $\tau \in[0, T)$, $u^{+}=\kappa(x), \tau^{+}=0$ if $\tau=T$. This represents that the state is sampled every $T$ units of time, after each sampling the control $u$ is computed based on the feedback $\kappa$, and in between sampling times, the computed constant control is applied. The variable $\tau$ is the timer.
} 
Following [5], we now make the concept of a solution to (2.2) precise. A subset $S \subset \mathbb{R}_{\geq 0} \times \mathbb{N}$ is a compact hybrid time domain if $S=\bigcup_{j=0}^{J-1}\left(\left[t_{j}, t_{j+1}\right], j\right)$ for some finite sequence of times $0=t_{0} \leq t_{1} \leq t_{2} \leq \ldots \leq t_{J}$. $S$ is a hybrid time domain if for all $(T, J) \in S, S \cap([0, T] \times\{0,1, \ldots, J\})$ is a compact hybrid domain; equivalently, if $S$ is a union of a finite or infinite sequence of intervals $\left[t_{j}, t_{j+1}\right] \times\{j\}$, with the last interval, if it exists, possibly of the form $\left[t_{j}, T\right)$ with $T$ finite or $T=+\infty$. We write $\sup _{t}(S)$ for the $\operatorname{supremum}$ of all $t$ such that $(t, j) \in S$ for some $j$.

A solution to the hybrid system (2.2) consists of: a nonempty hybrid time domain $S$, a function $x: S \rightarrow O$ such that $x(t, j)$ is locally absolutely continuous in $t$ for a fixed $j$ and constant in $j$ for a fixed $t$, where $(t, j) \in S$, and a function $q: S \rightarrow Q$ such that $q(t, j)$ is constant in $t$ for a fixed $j$, where $(t, j) \in S$, meeting the following conditions: $x(0,0) \in C_{q(0,0)} \cup D_{q(0,0)}$ and

(S1) for all $j \in \mathbb{N}$ and almost all $t$ such that $(t, j) \in S$,

$$
\dot{x}(t, j)=F_{q(t, j)}(x(t, j)), \quad x(t, j) \in C_{q(t, j)} ;
$$

(S2) for all $(t, j) \in S$ such that $(t, j+1) \in S$,

$$
q(t, j+1) \in G_{q(t, j)}(x(t, j)), \quad x(t, j) \in D_{q(t, j)} .
$$

Given a solution to (2.2), we will usually not mention the hybrid time domain explicitly, but will identify the solution by $(x, q)$. When needed, we will refer to the associated domain by $\operatorname{dom}(x, q)$.

The set $\mathcal{A}$ is stable for the hybrid system (2.2) if for any $\varepsilon>0$ there exists $\delta>0$ such that any solution $(x, q)$ to $(2.2)$ with $\operatorname{dist}_{\mathcal{A}}(x(0,0)) \leq \delta$ satisfies $\operatorname{dist}_{\mathcal{A}}(x(t, j)) \leq \varepsilon$ for all $(t, j) \in \operatorname{dom}(x, q)$. (Here and in what follows, $\operatorname{dist}_{\mathcal{A}}(x)=\min _{y \in \mathcal{A}}|x-y|$, where $|\cdot|$ is any chosen norm in $\mathbb{R}^{n}$.) The set $\mathcal{A}$ is globally attractive for the hybrid system (2.2) if for any $\left(x_{0}, q_{0}\right) \in \mathcal{O} \times Q$ there exists a solution to $(2.2)$ with $x(0,0)=x_{0}, q(0,0)=q_{0}$ and for any maximal solution $(x, q)$ to $(2.2)$ we have $\operatorname{dist}_{\mathcal{A}}(x(t, j)) \rightarrow 0$ as $t \rightarrow \sup _{t}(\operatorname{dom}(x, q))$. Finally, $\mathcal{A}$ is globally asymptotically stable for (2.2) if it is both stable and globally attractive.

By an admissible perturbation radius we will understand any continuous function $\rho: \mathcal{O} \rightarrow \mathbb{R}_{>0}$ such that $x+\rho(x) \mathbb{B} \subset \mathcal{O}$ for all $x \in \mathcal{O}$. (Here and in what follows, $\mathbb{B}$ is the closed unit ball in $\mathbb{R}^{n}$.) We will say that $\mathcal{A}$ is robustly globally asymptotically stable for (2.2) if there exists an admissible perturbation radius $\rho$ such that, for the hybrid system

$$
\begin{aligned}
& \dot{x} \in F_{q}^{\rho}(x) \quad \text { if } \quad x \in C_{q}^{\rho} \text {, } \\
& q^{+} \in G_{q}^{\rho}(x) \quad \text { if } \quad x \in D_{q}^{\rho} \text {, }
\end{aligned}
$$

with the data

$$
\begin{gathered}
F_{q}^{\rho}(x):=\operatorname{con}_{q}\left((x+\rho(x) \mathbb{B}) \cap C_{q}\right)+\rho(x) \mathbb{B} \\
G_{q}^{\rho}(x):=G_{q}\left((x+\rho(x) \mathbb{B}) \cap D_{q}\right), \\
C_{q}^{\rho}:=\left\{x \in O \mid(x+\rho(x) \mathbb{B}) \cap C_{q} \neq \emptyset\right\}, \\
D_{q}^{\rho}:=\left\{x \in O \mid(x+\rho(x) \mathbb{B}) \cap D_{q} \neq \emptyset\right\},
\end{gathered}
$$

the set $\mathcal{A}$ is globally asymptotically stable. (Above, con $F_{q}$ stands for the pointwise closed convex hull of $F_{q}$.) Such robustness can be understood as robustness to autonomous perturbations. When nonautonomous perturbations are considered, the very issue of the existence of solutions to the perturbed hybrid system is more delicate; see [9], Example 5.8. However, given a hybrid patchy feedback that leads to $\mathcal{A}$ being robustly globally asymptotically stable in the sense described above, a minor modification of the feedback guarantees existence of solutions under nonautonomous perturbations; see [9], Section V.C. 


\section{MAin RESUlt}

We call a control $u:[0, T] \rightarrow U$ piecewise constant if for some $0=t_{0}<t_{1}<t_{2}<\ldots<t_{P}=T, u(t)$ is constant for $t \in\left(t_{i-1}, t_{i}\right), i=1,2, \ldots, P$.

Theorem 3.1. Suppose that the function $x \mapsto f(x, u)$ is continuous and solutions to $\dot{x}(t)=f(x(t), u)$ are unique for each $u \in U$ and each initial condition in $\mathcal{O}$. Suppose furthermore that the nonlinear control system (2.1) is such that

(a) for each $\xi \in \mathcal{O}$ and $\gamma>0$ there exists $T_{\xi}>0$ and a piecewise constant $u_{\xi}:\left[0, T_{\xi}\right] \rightarrow U$ such that the trajectory $x_{\xi}$ to $(2.1)$ with $x_{\xi}(0)=\xi$ and $u$ replaced by $u_{\xi}$ exists on $\left[0, T_{\xi}\right]$ and $\operatorname{dist}_{\mathcal{A}}\left(x_{\xi}\left(T_{\xi}\right)\right)<\gamma$;

(b) for any $\varepsilon>0$ there exists $\delta>0$ such that for any $\xi \in \mathcal{O}$ with $\operatorname{dist}_{\mathcal{A}}(\xi)<\delta$ and any $\gamma>0$ one can find $u_{\xi}$ as in (a) and an associated $T_{\xi}>0$ so that the resulting trajectory $x_{\xi}$ is such that $\operatorname{dist}_{\mathcal{A}}\left(x_{\xi}(t)\right)<\varepsilon$ for all $t \in\left[0, T_{\xi}\right]$.

Then, there exists a strongly regular hybrid patchy feedback on $\mathcal{O}$ with $k_{q}$ constant for each $q \in Q$ that renders $\mathcal{A}$ asymptotically stable on $\mathcal{O}$ for the system (2.1).

We prove this result in Section 4. Here, we first note that assumptions (a) and (b) of Theorem 3.1 are met if (2.1) is asymptotically controllable to $\mathcal{A}$; that is, for each $\xi \in \mathcal{O}$, there exists a measurable and locally bounded $u_{\xi}:[0, \infty) \rightarrow U$ such that the resulting trajectory to $(2.1)$ satisfies $\operatorname{dist}_{\mathcal{A}}\left(x_{\xi}(t)\right) \rightarrow 0$ as $t \rightarrow \infty$, and if for any $\varepsilon>0$ there exists $\delta>0$ such that for $\xi \in \mathcal{O}$ with $\operatorname{dist}_{\mathcal{A}}(\xi)<\delta$, the said control $u_{\xi}$ can be found so that $\operatorname{dist}_{\mathcal{A}}\left(x_{\xi}(t)\right)<\varepsilon$ for all $t \geq 0$, and when $f$ is sufficiently regular to guarantee that the solutions to (2.1) are unique if measurable and locally bounded controls are applied. We add that the assumptions (a) and (b) are stated exactly in the form that will be used in the construction of the stabilizing feedback.

Second, let us explain the significance of the properties (regularity and strong regularity) of the stabilizing hybrid patchy feedback whose existence is claimed in Theorem 3.1. If the feedback is regular and $f$ is continuous (in both variables) then the resulting closed-loop hybrid system (2.2) has the properties required by [5] in developing the robust stability theory for hybrid systems. In particular, for such hybrid systems, appropriately understood limits of solutions are still solutions, and solutions from points near a reference point are close (again, in appropriate sense) to some solution from the reference point. Such properties guarantee that asymptotic stability of compact sets is always robust; see [5]. Technically, the robustness results of [5] is not applicable here, since we are talking about stability of $\mathcal{A} \times Q$, which need not be compact, and $f$ is not continuous in $(x, u)$. However, for such setting, if the hybrid feedback is not only regular, but strongly regular and $k_{q}$ is constant for each $q \in Q$, robustness of stability again comes for "free", thanks to [9], Theorem 4.3. Consequently:

Corollary 3.2. Under the assumptions of Theorem 3.1, there exists a strongly regular hybrid patchy feedback on $\mathcal{O}$ that renders $\mathcal{A}$ globally robustly asymptotically stable on $\mathcal{O}$ for the system (2.1).

\section{Stabilizing HybRid FeEdBack}

We now prove Theorem 3.1. In Section 4.1 a hybrid patchy feedback is constructed, in Section 4.2 basic properties of the feedback and of the resulting closed-loop hybrid system are described, and in Section 4.3 they are employed to conclude asymptotic stability of the closed loop.

A proper indicator of $\mathcal{A}$ with respect to $\mathcal{O}$ is any continuous function $\omega: \mathcal{O} \rightarrow(0, \infty)$ such that $\omega\left(\xi_{i}\right) \rightarrow 0$ if $\xi_{i} \rightarrow \mathcal{A}$, while $\omega\left(\xi_{i}\right) \rightarrow \infty$ if either $\xi_{i} \in \mathcal{O}$ converge to a boundary point of $\mathcal{O}$ not contained in $\mathcal{A}$ or $\left|\xi_{i}\right| \rightarrow \infty$. (Equivalently, $\omega$ is a restriction to $\mathcal{O}$ of a continuous function $\widetilde{\omega}: \widetilde{\mathcal{O}} \rightarrow[0, \infty)$ such that $\widetilde{\omega}(\xi)=0$ if and only if $\xi \in \mathcal{A}$ and $\widetilde{\omega}\left(\xi_{i}\right) \rightarrow \infty$ if either $\xi_{i} \in \widetilde{\mathcal{O}}$ converge to a boundary point of $\widetilde{\mathcal{O}}$ or $\left|\xi_{i}\right| \rightarrow \infty$.) Note that for any $0<r \leq r^{\prime}$, the set $\left\{\xi \in \mathcal{O} \mid r \leq \omega(\xi) \leq r^{\prime}\right\}$ is compact. 


\subsection{Construction}

Let $\omega: \mathcal{O} \rightarrow(0, \infty)$ be any proper indicator of $\mathcal{A}$ with respect to $\mathcal{O}$. Pick any $r_{0}>0$. Using assumption (b), find $r_{1}>0$ such that $r_{1}<r_{0} / 2$ and for each $\xi \in \mathcal{O}$ with $\omega(\xi) \leq r_{1}$ a piecewise constant $u_{\xi}:\left[0, T_{\xi}\right] \rightarrow U$ as in assumption (a) can be found (with any $\gamma>0$ ) so that the resulting trajectory $x_{\xi}$ satisfies $\omega(x(t))<r_{0}$ for all $t \in\left[0, T_{\xi}\right]$.

Fix $\xi \in R_{0}:=\left\{\xi \in \mathcal{O} \mid r_{1} \leq \omega(\xi) \leq r_{0}\right\}$. Find a piecewise constant $u_{\xi}:\left[0, T_{\xi}\right] \rightarrow U$ such that the resulting trajectory $x_{\xi}$ satisfies $\omega\left(x\left(T_{\xi}\right)\right)<r_{1}$ while for all $t \in\left[0, T_{\xi}\right], \omega(x(t))>r_{1} / 2$. Let $u_{\xi}$ be given by $u_{\xi}(t)=u_{\xi}^{m} \in U$ when $t \in\left(t_{\xi}^{m-1}, t_{\xi}^{m}\right)$, where

$$
0=t_{\xi}^{0}<t_{\xi}^{1}<t_{\xi}^{2}<\ldots<t_{\xi}^{M_{\xi}-1}<t_{\xi}^{M_{\xi}}=T_{\xi}
$$

For a point $\eta$ and a control value $u \in U$, let $\phi(\eta, u, t)$ be the trajectory of (2.1) with constant control $u$ starting at $\eta$ evaluated at time $t$ (so in particular, $\phi(\eta, u, 0)=\eta$ ). For a set $S$, a control $u \in U$, and an interval $[a, b]$, we write $\phi(S, u,[a, b])$ for $\bigcup_{\eta \in S} \bigcup_{t \in[a, b]} \phi(\eta, u, t)$.

Continuity of $f$ and the uniqueness of solutions assumption imply continuous dependence of solutions on initial conditions. In particular, for any $t^{\prime} \in\left[0, T_{\xi}\right]$ and any $\mu>0$ there exists $\mu^{\prime}>0$ (which can be chosen arbitrarily small) so that any solution to $\dot{x}(t)=f\left(x(t), u_{\xi}(t)\right)$ with $x(0) \in x_{\xi}\left(t^{\prime}\right)+\mu^{\prime} \mathbb{B}$ is such that $x(t) \in x_{\xi}\left(t^{\prime}+t\right)+\mu \mathbb{B}$ for all $t \in\left[0, T_{\xi}-t^{\prime}\right]$. Consequently, one can pick $\varepsilon_{\xi}^{m}>0, \delta_{\xi}^{m}>\varepsilon_{\xi}^{m}, m=0,1, \ldots, M_{\xi}-1$ so that the closed sets

$$
\phi\left(x_{\xi}\left(t_{\xi}^{m}\right)+\delta_{\xi}^{m} \mathbb{B}, u_{\xi}^{m+1},\left[0, t_{\xi}^{m+1}-t_{\xi}^{m}\right]\right)
$$

are bounded (hence compact) and such that

$$
\begin{gathered}
\phi\left(x_{\xi}\left(t_{\xi}^{m}\right)+\delta_{\xi}^{m} \mathbb{B}, u_{\xi}^{m+1}, t_{\xi}^{m+1}-t_{\xi}^{m}\right) \subset \operatorname{int}\left(x_{\xi}\left(t_{\xi}^{m+1}\right)+\varepsilon_{\xi}^{m+1} \mathbb{B}\right), \\
\inf \omega\left(\phi\left(x_{\xi}\left(t_{\xi}^{m-1}\right)+\delta_{\xi}^{m-1} \mathbb{B}, u_{\xi}^{m},\left[0, t_{\xi}^{m}-t_{\xi}^{m-1}\right]\right)\right)>r_{1} / 2
\end{gathered}
$$

for $m=0,1, \ldots, M_{\xi}-1$, while

$$
\sup \omega\left(\phi\left(x_{\xi}\left(t_{\xi}^{M_{\xi}-1}\right)+\delta_{\xi}^{M_{\xi}-1} \mathbb{B}, u_{\xi}^{M_{\xi}}, t_{\xi}^{M_{\xi}}-t_{\xi}^{M-1}\right)\right)<r_{1}
$$

The procedure above can be repeated for each $\xi \in R_{0}$. Since $R_{0}$ is compact, one can find $\xi_{1}, \xi_{2}, \ldots, \xi_{L_{0}} \in R_{0}$ so that

$$
\bigcup_{l=1}^{L_{0}} \xi_{l}+\varepsilon_{\xi_{l}}^{0} \operatorname{int} \mathbb{B} \supset R_{0} .
$$

For $l=1,2, \ldots, L_{0}, m=1,2, \ldots, M_{\xi_{l}}$, define the control values

$$
u_{0, l, m}=u_{\xi_{l}}^{m}
$$

and sets

$$
\begin{aligned}
& \Theta_{0, l, m}=\phi\left(x_{\xi_{l}}\left(t_{\xi_{l}}^{m-1}\right)+\varepsilon_{\xi_{l}}^{m-1} \mathbb{B}, u_{\xi_{l}}^{m},\left[0, t_{\xi_{l}}^{m}-t_{\xi_{l}}^{m-1}\right]\right), \\
& \Theta_{0, l, m}^{\prime}=\phi\left(x_{\xi_{l}}\left(t_{\xi_{l}}^{m-1}\right)+\delta_{\xi_{l}}^{m-1} \mathbb{B}, u_{\xi_{l}}^{m},\left[0, t_{\xi_{l}}^{m}-t_{\xi_{l}}^{m-1}\right]\right) .
\end{aligned}
$$

These subsets of $\mathcal{O}$ are compact and such that $\Theta_{0, l, m}$ is a subset of the interior of $\Theta_{0, l, m}^{\prime}$. Note also that since $\xi_{l}+\varepsilon_{\xi_{l}}^{0} \mathbb{B} \subset \Theta_{0, l, 1}$, the sets $\Theta_{0, l, m}$ (and thus also the sets $\Theta_{0, l, m}^{\prime}$ ) form a covering of $R_{0}$. 
Now proceed inductively. Given $r_{k+2}$ and $r_{k+1}, k=-1,-2,-3, \ldots$, controls $u_{k, l, m}$, and sets $\Theta_{k, l, m}, \Theta_{k, l, m}^{\prime}$, $l=1,2, \ldots, L_{k}, m=1,2, \ldots, M_{\xi_{l}}$, pick $r_{k}$ so that $r_{k}>2 r_{k+1}$ and

$$
r_{k}>\max \omega\left(\bigcup_{l=1}^{L_{k+1}} \bigcup_{m=1}^{M_{\xi_{l}}} \Theta_{k+1, l, m}^{\prime}\right)
$$

and define $R_{k}:=\left\{\xi \in \mathcal{O} \mid r_{k+1} \leq \omega(\xi) \leq r_{k}\right\}$. Repeat the construction (carried out above for $R_{0}$ ) for $R_{k}$, starting by finding, for each $\xi \in R_{k}$, a piecewise constant $u_{\xi}:\left[0, T_{\xi}\right] \rightarrow U$ such that the resulting trajectory $x_{\xi}$ satisfies $\omega\left(x\left(T_{\xi}\right)\right)<r_{k+1}$ while for $t \in\left[0, T_{\xi}\right], \omega(x(t))>r_{k+1} / 2$.

Similarly, given $r_{k}$ and $r_{k-1}, k=1,2,3, \ldots$, pick $r_{k+1}$ so that $r_{k+1}<r_{k} / 2$ and for any $\xi \in \mathcal{O}$ with $\omega(\xi) \leq r_{k+1}$ (and any $\gamma>0$ ) one can find $u_{\xi}:\left[0, T_{\xi}\right] \rightarrow U$ so that the resulting trajectory $x_{\xi}$ is such that $\omega\left(x_{\xi}(t)\right)<r_{k}$ for all $t \in\left[0, T_{\xi}\right]$. Define $R_{k}:=\left\{\xi \in \mathcal{O} \mid r_{k+1} \leq \omega(\xi) \leq r_{k}\right\}$. Repeat the construction (carried out above for $R_{0}$ ) for $R_{k}$, starting by finding, for each $\xi \in R_{k}$, a piecewise constant $u_{\xi}:\left[0, T_{\xi}\right] \rightarrow U$ such that the resulting trajectory $x_{\xi}$ satisfies $\omega\left(x\left(T_{\xi}\right)\right)<r_{k+1}$ while for $t \in\left[0, T_{\xi}\right], \omega(x(t))>r_{k+1} / 2$.

In this fashion, one obtains numbers $r_{k}, k \in \mathbb{Z}$, such that $r_{k}<r_{k-1} / 2$ and a family of controls $u_{k, l, m}$ and sets $\Theta_{k, l, m}, \Theta_{k, l, m}^{\prime}, k \in \mathbb{Z}, l=1,2, \ldots, L_{k}, m=1,2, \ldots, M_{\xi_{l}}$, such that, in particular,

$$
r_{k+2}<r_{k+1} / 2<\omega\left(\Theta_{k, l, m}^{\prime}\right)<r_{k-1}
$$

and thus the covering of $\mathcal{O}$ by sets $\Theta_{k, l, m}^{\prime}$ (and thus also by the sets $\Theta_{k, l, m}$ ) is locally finite.

Let $Q^{\prime}$ be the (countable) family of triples $k, l, m, k \in \mathbb{Z}, l=1,2, \ldots, L_{k}, m=1,2, \ldots, M_{\xi_{l}}$, ordered as follows: if $q=(k, l, m)$ and $q^{\prime}=\left(k^{\prime}, l^{\prime}, m^{\prime}\right)$, then

$$
q \succ q^{\prime} \quad \text { if } \quad k>k^{\prime} \text { or } k=k^{\prime}, l>l^{\prime} \text { or } k=k^{\prime}, l=l^{\prime}, m>m^{\prime} .
$$

Let $Q \subset Q^{\prime}$ be the set of those $q$ for which $\Theta_{q}^{\prime} \backslash \bigcup_{q^{\prime} \succ q} \Theta_{q^{\prime}} \neq \emptyset$.

Define the hybrid feedback as follows: for each $q \in Q$, let

$$
\begin{gathered}
C_{q}=\overline{\Theta_{q}^{\prime} \backslash \bigcup_{q^{\prime} \succ q} \Theta_{q^{\prime}}}, \quad D_{q}=\left(\bigcup_{q^{\prime} \succ q} \Theta_{q^{\prime}}\right) \cup \overline{\mathcal{O} \backslash \Theta_{q}^{\prime}}, \\
k_{q}(\xi)=u_{q}, \\
G_{q}(\xi)=\left\{\begin{array}{cc}
\left\{q^{\prime} \in Q \mid \xi \in \Theta_{q^{\prime}}\right\} & \xi \in \overline{\mathcal{O} \backslash \Theta_{q}^{\prime}} \\
\left\{q^{\prime} \in Q \mid \xi \in \Theta_{q^{\prime}}, q^{\prime} \succ q\right\} & \xi \in\left(\bigcup_{q^{\prime} \succ q} \Theta_{q^{\prime}}\right) \backslash \overline{\mathcal{O} \backslash \Theta_{q}^{\prime}}
\end{array}\right.
\end{gathered}
$$

Here and in what follows, the closure operation is taken relative to the open set $\mathcal{O}$. (This in particular ensures that $\overline{\mathcal{O} \backslash \Theta_{q}^{\prime}} \subset \mathcal{O}$, and so $D_{q} \subset \mathcal{O}$.) In closed loop with (2.1), this feedback leads to a hybrid system given by $C_{q}, D_{q}, G_{q}$ as above and $F_{q}$ given by $F_{q}(\xi)=f\left(\xi, u_{q}\right)$.

\subsection{Properties of the hybrid system and of the solutions}

We now make several observations about the structure of the constructed hybrid feedback (4.3) and the resulting closed-loop hybrid system (2.2).

By construction, given $q=(k, l, m) \in Q^{\prime}$, we have $\Theta_{k, l, m}^{\prime} \subset R_{k-1} \cup R_{k} \cup R_{k+1}$. Since for each $k$ there is finitely many $q=(k, l, m) \in Q^{\prime}$, only finitely many sets $\Theta_{q}^{\prime}$ intersect each $R_{k}$, which in turn implies that each compact subset of $\mathcal{O}$ intersects finitely many $\Theta_{q}^{\prime}$. The same conclusion holds for $\Theta_{q}$ 's, as $\Theta_{q} \subset \Theta_{q}^{\prime}$. By construction, 
for each $\xi \in \mathcal{O}$ there exists some $\Theta_{q}$ such that $\xi \in \Theta_{q}$. (In fact, if $\xi \in R_{k}$, for some $l$ such that $(k, l, 1) \in Q^{\prime}$ we have $\xi \in \Theta_{(k, l, 1)}$.) So, $\left\{\Theta_{q}\right\}_{q \in Q}$ and also $\left\{\Theta_{q}^{\prime}\right\}_{q \in Q}$ form locally finite coverings of $\mathcal{O}$. Consequently, for each $\xi \in \mathcal{O}$ there exists "the largest" index $q$ such that $\xi \in \Theta_{q}$; we will write

$$
q^{*}(\xi):=\max \left\{q \in Q \mid \xi \in \Theta_{q}\right\}
$$

Thus for any $\xi \in \mathcal{O}$,

$$
\xi \in \Theta_{q^{*}(\xi)} \backslash \bigcup_{q \succ q^{*}(\xi)} \Theta_{q} \subset \Theta_{q^{*}(\xi)}^{\prime} \backslash \bigcup_{q \succ q^{*}(\xi)} \Theta_{q} \subset C_{q^{*}(\xi)} .
$$

This means that $\left\{C_{q}\right\}_{q \in Q}$ covers $\mathcal{O}$. That it is a locally finite covering follows from the fact that $C_{q} \subset \Theta_{q}^{\prime}$, and the covering by $\Theta_{q}^{\prime}$ 's is locally finite.

Lemma 4.1. The hybrid feedback (4.3) is strongly regular.

Proof. By construction, $Q \subset \mathbb{Z}^{3}$, and $Q$ is totally ordered by (4.2). For each $q \in Q, \Theta_{q}$ and $\Theta_{q}^{\prime}$ are closed (not just relatively closed) subsets of $\mathcal{O}$. Thus, $C_{q}$ is a closed (not just relatively closed) subset of $\mathcal{O}$, while local finiteness of the family $\left\{\Theta_{q}\right\}_{q \in Q}$ leads to $D_{q}$ being a relatively closed subset of $\mathcal{O}$. Mappings $k_{q}$ are constant by definition. Mappings $G_{q}$ map to $\left\{q^{\prime} \in Q \mid \xi \in \Theta_{q^{\prime}}\right\}$, and thus are locally bounded uniformly in $q$, since the covering of $\mathcal{O}$ by $\Theta_{q^{\prime}}$ 's is locally finite. That $\Theta_{q^{\prime}}$ 's actually cover $\mathcal{O}$ implies that $G_{q}(\xi)$ is nonempty when $\xi \in D_{q}$. To see that each $G_{q}$ is outer semicontinuous on $\mathcal{O}$, take a convergent sequence $\xi_{i} \in D_{q}$ and a convergent (and hence eventually constant) sequence $q_{i} \in G_{q}\left(\xi_{i}\right)$. Let the corresponding limits be, respectively, $\xi$ and $q^{\prime}$. Then for all large enough $i, \xi_{i} \in \Theta_{q^{\prime}}$, and since $\Theta_{q^{\prime}}$ is closed, $\xi \in \Theta_{q^{\prime}}$. This implies that $q^{\prime} \in G_{q}(\xi)$ if $\xi \in \overline{\mathcal{O} \backslash \Theta_{q}^{\prime}}$. If $\xi \notin \overline{\mathcal{O} \backslash \Theta_{q}^{\prime}}$, then $\xi_{i} \notin \overline{\mathcal{O} \backslash \Theta_{q}^{\prime}}$ for all large enough $i$, and thus $q^{\prime} \succ q$. This now yields that $q^{\prime} \in G_{q}(\xi)$. In the paragraph above the lemma, we have already argued that $C_{q}$ 's form a locally finite covering of $\mathcal{O}$. Finally, it is straightforward to verify that $C_{q} \cup D_{q}=\mathcal{O}$.

Since for each $q \in Q, C_{q} \cup D_{q}=\mathcal{O}$, nontrivial solutions to (2.2) exist for any initial point, and each maximal solution $(x, q)$ (that is, a solution that cannot be extended) is either complete (that is, has unbounded domain), "blows up" in finite hybrid time, or approaches the boundary of $\mathcal{O} \times Q$ in finite hybrid time (which means that it is either complete, or for (a finite) $(T, J)=\sup \operatorname{dom}(x, q),|x(t, J)|$ diverges to $\infty$ or $x(t, J)$ approaches the boundary of $\mathcal{O}$ as $t \rightarrow T$ ). See [5]. Since the covering of $\mathcal{O}$ by $C_{q}$ 's is locally finite, the finite time "blow up" or approaching the boundary of $\mathcal{O}$ implies that $(x, q)$ jumps infinitely many times, and thus is complete anyway. Consequently, each maximal solution $(x, q)$ is complete. (This does not mean that $x$ exists on $[0, \infty)$, in fact, it may happen that $x$ reaches $\mathcal{A}$ in finite time.)

Note that each maximal solution jumps at least once (and thus infinitely many times). Indeed, otherwise there exists a complete solution (i.e. $x$ is defined on $[0, \infty))$ to $\dot{x}(t)=f\left(x(t), u_{q}\right), x(t) \in C_{q}$, for some $q \in Q$. However, by construction, each solution to $\dot{x}(t)=f\left(x(t), u_{q}\right)$ starting in $C_{q}$ (and so in $\Theta_{k, l, m}^{\prime}$ for some triple $k, l, m$ ) in finite time reaches a point, say $\xi$, in the interior of either $\Theta_{k, l, m+1}$ or of some $\Theta_{k+1, l^{\prime}, 0}$. Either way, $q^{*}(\xi) \succ q$, and such a solution enters the interior of $\Theta_{q^{\prime}}$ for some $q^{\prime} \succ q$, and thus leaves $C_{q}$. This is a contradiction.

Finally, note that the set

$$
\bigcup_{q \in Q} \overline{\Theta_{q} \backslash \bigcup_{q^{\prime} \succ q} \Theta_{q^{\prime}}} \times\{q\}
$$

is forward invariant, and that each solution to (2.2) enters that set after its first jump. Indeed, a solution $x$ to $\dot{x}(t)=\left(f\left(x(t), u_{q}\right), x(t) \in C_{q}\right.$ starting in $\Theta_{q}$ remains in $\Theta_{q}$ (since this set was defined as a reachable set) until it reaches $\bigcup_{q^{\prime} \succ q} \Theta_{q^{\prime}}$. Also, for any $q \in Q$ and $\xi \in D_{q},\left(\xi, G_{q}(\xi)\right)$ is a subset of the set (4.4), by the definition of $G_{q}$. A particular consequence of the invariance is that, after the first jump, the discrete variable of any solution is increasing at each jump. Thus, by the local finiteness of the covering by $\Theta_{q}$ 's, there are no instantaneous Zeno solutions (solutions with domains given by $0 \times \mathbb{N}$ ). 


\subsection{Asymptotic stability}

The properties of the solutions to the hybrid system (2.2), described in Section 4.2, are now employed to deduce that $\mathcal{A}$ is asymptotically stable for (2.2).

Stability. Let $(x, q)$ be a solution with $\omega(x(0,0)) \leq r_{i}$ for some $i \in \mathbb{Z}$. Suppose $(0,1) \in \operatorname{dom}(x, q)$ and let $q(0,1)=(k, l, m)$. Then $x(0,0) \in \Theta_{k, l, m}$ and so $k \geq i-1$ by (4.1). Since $q$ is nondecreasing "after the first jump", as argued following $(4.4), q(t, j)=\left(k^{\prime}, l^{\prime}, m^{\prime}\right)$ satisfies $k^{\prime} \geq i-1$ for all $(t, j) \in \operatorname{dom}(x, q), j \geq 1$. As for all such $(t, j), x(t, j) \in \Theta_{q(t, j)}$, inequality (4.1) implies that $\omega(x(t, j)) \leq r_{i-2}$. Now suppose that $(t, 0) \in \operatorname{dom}(x, q)$ for some $t>0$. Then $\omega(x(t, 0)) \leq r_{i-1}$ for all $t$ such that $(t, 0) \in \operatorname{dom}(x, q)$, by the construction of $r_{i-1}$. Arguments given above, for the case of $(0,1) \in \operatorname{dom}(x, q)$ can be now applied "after the first jump". Then $\omega(x(t, j)) \leq r_{i-3}$ for all $(t, j) \in \operatorname{dom}(x, q)$.

Attractivity. Any maximal solution $(x, q)$ jumps infinitely many times, as argued above (4.4), and after the first jump, $q$ increases at each jump. This, the finiteness of the covering of $\mathcal{O}$ by $\Theta_{q}$ 's, and stability shown above, implies that $x(t, j) \rightarrow \mathcal{A}$ as $(t, j) \rightarrow \sup \operatorname{dom}(x, q)$.

This concludes the proof of Theorem 3.1.

\section{Relation to PATChy FEEDBACK}

In [1], under stronger assumptions, it was shown that the nonlinear system (2.1) can be stabilized with a patchy feedback. (Technically, [1] deals with the case of $\widetilde{\mathcal{O}}=\mathbb{R}^{n}$ and $\mathcal{A}=\{0\}$; an extension to the more general case of any open $\widetilde{\mathcal{O}}$ and compact $\mathcal{A} \subset \widetilde{\mathcal{O}}$ is straightforward, as noted in [9].) A patchy feedback is a mapping $\Phi: \mathcal{O} \rightarrow U$ that can be written as

$$
\Phi(\xi)=u_{\alpha} \quad \text { if } \quad \xi \in \Omega_{\alpha} \backslash \bigcup_{\beta \succ \alpha} \Omega_{\beta},
$$

where indices $\alpha$ (and $\beta$ ) are in some totally ordered index set, $u_{\alpha} \in U$, sets $\Omega_{\alpha}$ form a locally finite covering of $\mathcal{O}$, and each of these sets is a patch (which entails $\Omega_{\alpha}$ being open, and that solutions to $\dot{x}=f\left(x, u_{\alpha}\right)$ from the boundary of $\Omega_{\alpha}$ immediately flow into $\left.\Omega_{\alpha}\right)$. Solutions to the resulting closed-loop system $\dot{x}=f(x, \Phi(x))$ are understood in the Caratheodory sense (and the system is a continuous-time differential equation with discontinuous right-hand side, not a hybrid system). Patchy feedbacks of [1] are robust to external disturbances, and in a semiglobal practical sense, to measurement error of bounded variation, as shown in [2]. They can be made hybrid to achieve robustness to general measurement error; this was done in [9].

Here, as a byproduct of the construction of a hybrid feedback, we can also construct a "patchy-like" feedback, leading to a continuous-time closed-loop system with a discontinuous right-hand side. However, that "patchylike" feedback does not enjoy the good properties of the patchy feedback of [1]. More specifically, given the (closed) sets $\Theta_{q}, q \in Q$, as constructed in Section 4.1, consider $\Psi: \mathcal{O} \rightarrow U$ given by

$$
\Psi(\xi)=u_{q}, \quad \text { if } \quad \xi \in \Theta_{q} \backslash \bigcup_{q^{\prime} \succ q} \Theta_{q^{\prime}} .
$$

Arguments very similar to those in Sections 4.2 and 4.3 can be used to show that $\Psi$ renders $\mathcal{A}$ asymptotically stable. Several desired properties present for the patchy feedback $\Phi$ are not enjoyed by $\Psi$. For example, pointwise limits of solutions to $\dot{x}=f(x, \Phi(x))$ need not be solutions to that system: one can envision a situation where a sequence of solutions in a set $\Theta_{q}$ converge to an arc in $\Theta_{q^{\prime}}$, and the limiting arc fails to be a solution (this is caused by $\Theta_{q}$ 's being closed, in contrast to open patches $\Omega_{\alpha}$ ). Similarly, uniform limits of Euler solutions generated with decreasing initial measurement noise and external disturbances need not yield solutions to the system. Finally, arbitrarily small external disturbances can destroy the asymptotic stability: for example, small disturbances can cause solutions to flow out of $\Theta_{q}$ before they reach $\bigcup_{q^{\prime} \succ q} \Theta_{q^{\prime}}$.

In summary, the hybrid patchy feedback constructed in this paper has better robustness properties than the patchy feedback of [1]. These properties are achieved not via a different construction, but by passing to 
a hybrid framework - it does not appear that similar robustness levels are reachable in a non-hybrid discontinuous feedback setting. While a hybrid patchy feedback can be obtained by making the patchy feedback of [1] hybrid, as was done in [9], we construct it here directly, without the intermediate step of obtaining a patchy feedback first. The direct construction does lead, as a byproduct, to a "patchy-like" discontinuous feedback, but that feedback does not have any robustness properties.

\section{REFERENCES}

[1] F. Ancona and A. Bressan, Patchy vector fields and asymptotic stabilization. ESAIM: COCV 4 (1999) 445-471.

[2] F. Ancona and A. Bressan, Flow stability of patchy vector fields and robust feedback stabilization. SIAM J. Control Optim. 41 (2003) 1455-1476.

[3] F.H. Clarke, Y.S. Ledyaev, E.D. Sontag and A.I. Subbotin, Asymptotic controllability implies feedback stabilization. IEEE Trans. Automat. Control 42 (1997) 1394-1407.

[4] F.H. Clarke, Y.S. Ledyaev, L. Rifford and R.J. Stern, Feedback stabilization and Lyapunov functions. SIAM J. Control Optim. 39 (2000) $25-48$.

[5] R. Goebel and A.R. Teel, Solutions to hybrid inclusions via set and graphical convergence with stability theory applications. Automatica 42 (2006) 573-587.

[6] R. Goebel, C. Prieur and A.R. Teel, Smooth patchy control Lyapunov functions, in Proc. 45th IEEE Conference on Decision and Control (2006).

[7] C. Prieur, Perturbed hybrid systems, applications in control theory, in Nonlinear and adaptive control, A. Zinober and D. Owens Eds., Lecture Notes in Control and Information Sciences 281, Springer, Berlin (2003) 285-294.

[8] C. Prieur, Asymptotic controllability and robust asymptotic stabilizability. SIAM J. Control Optim. 43 (2005) $1888-1912$.

[9] C. Prieur, R. Goebel and A.R. Teel, Hybrid feedback control and robust stabilization of nonlinear systems. IEEE Trans. Automat. Control 52 (2007) 2103-2117.

[10] R.T. Rockafellar and R.J.-B. Wets, Variational Analysis. Springer (1998).

[11] E.D. Sontag, Clocks and insensitivity to small measurement errors. ESAIM: COCV 4 (1999) 537-557. 